\title{
Le syndrome de fatigue chronique et la fibromyalgie au Canada : prévalence et associations avec six indicateurs de l'état de santé
}

\author{
C. Rusu, M.D. (1); M. E. Gee, M. Sc. (1); C. Lagacé, M. Sc. (1); M. Parlor, L.L. B. (2)
}

Cet article a fait l'objet d'une évaluation par les pairs.

Diffuser cet article sur Twitter

\section{Résumé}

Introduction : Peu d'études ont traité, à l'aide de données populationnelles, des facteurs associés de façon indépendante au syndrome de fatigue chronique (SFC) et à la fibromyalgie (FM) ou des répercussions de ces affections sur l'état de santé.

Méthodologie : Nous avons utilisé les données de l'Enquête sur la santé dans les collectivités canadiennes de 2010 ( $n=59$ 101), représentative de la population à l'échelle nationale, pour décrire les cas autodéclarés de SFC et de FM diagnostiqués par un professionnel de la santé et pour déterminer les associations de ces affections avec six indicateurs de l'état de santé.

Résultats : En 2010, 1,4\% (intervalle de confiance [IC] à $95 \%: 1,3 \%$ à 1,6\%) des Canadiens de 12 ans ou plus vivant à domicile ont déclaré avoir reçu un diagnostic de SFC, $1,5 \%$ (IC à $95 \%: 1,4 \%$ à $1,7 \%$ ) de FM, et $0,3 \%$ (IC à $95 \%: 0,3 \%$ à $0,4 \%$ ) a déclaré être atteinte à la fois de SFC et de FM. Les cas de SFC comme ceux de FM étaient plus fréquents chez les femmes, les adultes de 40 ans ou plus, les personnes à faible revenu et les personnes présentant certains facteurs de risque de maladie chronique (obésité, sédentarité et tabagisme). Après ajustement en fonction des différences existant entre les groupes, les personnes ayant déclaré être atteintes du SFC ou de FM ou des deux avaient un moins bon état de santé que les personnes atteintes d'aucune de ces affections pour cinq indicateurs de l'état de santé, mais aucune différence n’a été trouvée entre ces groupes par rapport à l'indicateur de santé mentale. Le fait d'être atteint à la fois du SFC et de FM et de présenter de multiples affections comorbides était associé à un moins bon état de santé.

Conclusion : La présence concomitante du SFC, de la FM et d'autres affections chroniques était étroitement associée au fait d'avoir un moins bon état de santé, et les différences relatives à l'état de santé étaient dues en bonne partie à la présence concomitante de ces affections. La compréhension des facteurs qui contribuent à l'amélioration de la qualité de vie des personnes atteintes du SFC ou de FM, et en particulier des personnes qui présentent ces deux affections ainsi que diverses affections comorbides, serait un champ important à explorer dans le cadre de travaux de recherche ultérieurs.

Mots-clés : encéphalomyélite myalgique, fibromyalgie, état de santé, enquêtes sur la santé, études transversales

\section{Introduction}

En 2003, environ 1,3\% de la population canadienne d'âge adulte a déclaré être atteinte du syndrome de fatigue chronique (SFC), et environ $1,5 \%$ de fibromyalgie $(F M)^{1}$. Le SFC, que l'on appelle aussi encéphalomyélite myalgique, est caracté- risé par une fatigue physique et cognitive persistante et lourde, tandis que la FM est caractérisée par une douleur musculosquelettique chronique et généralisée ${ }^{2}$. De plus, ces deux affections se manifestent souvent en concomitance ${ }^{1-4}$. La présence concomitante de multiples affections chroniques chez une même personne accroît les coûts et l'utilisation des ressources du système de santé $^{5,6}$ et, comme on l'a montré avec d'autres affections chroniques, elle peut avoir une incidence considérable sur la qualité de vie liée à la santé ${ }^{6-10}$.

Quelques études menées au Canada ${ }^{1,2}$ et ailleurs $^{11-13}$ ont traité de l'incidence du SFC et de la FM sur l'état de santé. Lavergne et collab. $^{2}$ ont montré que les patients canadiens atteints du SFC ou de FM avaient une moins bonne santé (évaluée au moyen du formulaire abrégé SF-36) que la population canadienne en général. Chez cette population de patients fréquentant un établissement de soins tertiaires ou une clinique spécialisée, patients que les auteurs considèrent comme étant plus atteints que les autres personnes du même sexe et du même groupe d'âge présentant ces affections (p. ex. les personnes atteintes du SFC ou de FM choisies dans le cadre d'enquêtes populationnelles générales), la gravité de l'incapacité était associée à l'apparition des symptômes à un jeune âge, à un faible statut socioéconomique et à la présence concomitante du SFC et de la $\mathrm{FM}^{2}$. Néanmoins, les données de l'Enquête sur la santé dans les collectivités canadiennes (ESCC) de 2003, une enquête représentative de la population à l'échelle nationale, révèlent que les Canadiens atteints du SFC et de FM ont 
une moins bonne santé générale et mentale, sont moins satisfaits de leur vie, souffrent davantage de maladie mentale, ont besoin de plus d'aide pour accomplir certaines activités de la vie quotidienne et ont plus souvent recours à des services de santé ${ }^{1}$. Ces données ont également révélé une association entre le fait d'être une femme, d'être relativement âgé, d'avoir un faible revenu et d'être faiblement scolarisé et le fait d'être atteint du $\mathrm{SFC}^{1}$ et de $\mathrm{FM}^{1,14}$. Cependant, dans ces analyses, on ne cherchait pas à savoir si ces facteurs étaient associés de façon indépendante à ces affections.

En utilisant des données plus récentes, celles de l'ESCC de 2010, nous avons cherché à déterminer les facteurs associés de façon indépendante au fait d'être atteint du SFC et de FM, l'impact de ces affections sur l'état de santé et enfin les facteurs associés à un moins bon état de santé chez les Canadiens qui vivent avec ces affections.

\section{Méthodologie}

\section{Source des données}

Nous avons analysé des données du fichier partagé de la composante annuelle de l'ESCC de 2010. L'ESCC est une enquête transversale menée par Statistique Canada visant à recueillir des données sur la santé des Canadiens (état de santé, comportements relatifs à la santé, affections chroniques, déterminants individuels et socioéconomiques de la santé, etc.). La population ciblée était âgée de 12 ans ou plus et vivait en logement privé dans une province ou un territoire du Canada. L'enquête ne tenait pas compte des populations vivant en établissement, des membres à temps plein des Forces armées canadiennes ou encore des personnes vivant dans une réserve indienne, sur des terres publiques ou dans certaines régions éloignées, ce qui représente moins de $2 \%$ de la population canadienne de 12 ans ou plus. Les données ont été recueillies entre janvier et décembre 2010. On trouvera des précisions supplémentaires sur la méthodologie de l'enquête, notamment en ce qui concerne les stratégies visant à assurer la représentativité de l'échantillon, dans une publication antérieure ${ }^{15}$. Le taux de réponse global de l'enquête a été de
80,7 \% à l'échelle des ménages et de 88,6 \% à l'échelle individuelle, l'échantillon final étant composé des 59302 personnes de 12 ans ou plus ayant consenti à ce que leurs données soient divulguées à certains partenaires gouvernementaux.

\section{Stratégie analytique}

Nous avons élaboré notre stratégie analytique en trois étapes interreliées. Dans un premier temps, nous avons identifié des covariables à priori en nous fondant sur des études antérieures sur le SFC et la FM, en utilisant soit les données de l'ESCC ${ }^{1,14}$, soit des données recueillies dans un contexte clinique $^{2,3}$. Certaines covariables potentielles n'ont pas été prises en considération, à savoir la gravité de la maladie, la durée de la maladie et les événements stressants de la vie ${ }^{2}$, car elles n'avaient pas été mesurées dans l'ESCC. Dans un deuxième temps, nous avons examiné les relations bivariées entre des covariables potentielles et le SFC et la FM. Dans un troisième temps, les covariables pour lesquelles nous avons observé une relation bivariée avec le SFC et la FM ont été retenues dans un modèle multivarié. Notre stratégie analytique était limitée par la taille de l'échantillon. Afin d'obtenir une estimation fiable de la plupart des variables et covariables indicatrices de l'état de santé, il a fallu fusionner certaines catégories de réponses et dichotomiser certaines variables. Les sections suivantes décrivent en détail la façon dont chacune des variables a été analysée.

\section{SFC et FM}

Au cours de l'entretien, les deux questions suivantes étaient posées aux répondants : "Souffrez-vous du syndrome de fatigue chronique? » et "Souffrez-vous de fibromyalgie?». L'introduction suivante était lue aux répondants au début du module sur les problèmes de santé chroniques : "Les questions qui suivent portent sur certains problèmes de santé de longue durée que vous pouvez avoir. Par "problème de santé de longue durée”, on entend un état qui dure ou qui devrait durer 6 mois ou plus et qui a été diagnostiqué par un professionnel de la santé. » On considérait les répondants comme des cas s'ils répondaient « oui » à l'une ou l'autre de ces questions. Aucune vérification n'a été faite pour confirmer le diagnostic ou déterminer quelle définition de cas avait été utilisée par le professionnel de la santé ayant posé le diagnostic.

Les personnes ayant refusé ou s'étant abstenues de répondre aux questions sur le SFC ou la FM ont été exclues ( $\mathrm{n}=201)$, ce qui laissait 59101 répondants pour l'analyse.

\section{Covariables}

La prévalence du SFC comme de la FM ont été décrites en fonction du sexe, de l'âge (12 à 39 ans, 40 à 59 ans, 60 ans ou plus), de l'origine ethnique (Blanc, Autochtone, autre), du niveau de scolarité le plus élevé au sein du ménage (diplôme d'études postsecondaires, études post-secondaires partielles, diplôme d'études secondaires, études secondaires non terminées), de l'état matrimonial (célibataire, veuf(ve)/séparé(e)/ divorcé(e), marié(e)/conjoint(e) de fait) et du quintile de suffisance du revenu corrigé. En ce qui concerne ce dernier point, les répondants ont été répartis en quintiles en fonction du rapport entre le revenu total de leur ménage et le seuil de faible revenu correspondant à la taille de leur ménage et celle de leur collectivité tel qu'il a été déterminé par Statistique Canada. Cela a ainsi permis d'obtenir une mesure relative du revenu du ménage de chaque répondant comparable avec celle du revenu du ménage des autres répondants ${ }^{15}$.

En ce qui concerne le niveau de scolarité, nous avons ajouté une catégorie " non précisé » car $8 \%$ des participants n'avaient pas répondu à la question.

Lorsqu'il manque des renseignements sur le revenu des répondants, Statistique Canada effectue une imputation par le plus proche voisin, laquelle modélise le revenu en fonction de la structure familiale, de données sociodémographiques, de certaines variables liées à la santé et du revenu correspondant aux renseignements fiscaux. C'est ainsi que le revenu a été déterminé pour $33 \%$ des répondants (18\% à partir de revenus entièrement déclarés, $4 \%$ à partir de revenus partiellement déclarés et $12 \%$ sans information sur les revenus $)^{15}$. Nous avons également conçu une catégorie « non précisé » pour les 2,4\% de répondants pour lesquels il manquait certaines données quant à la variable du revenu, soit des résidents des trois territoires, car Statistique Canada ne 
calcule pas de quintile de suffisance du revenu corrigé pour ces résidents.

La prévalence du SFC et celle de la FM ont également été décrites en fonction de l'indice de masse corporelle (IMC), d'après la taille et le poids autodéclarés (poids insuffisant ou normal : moins de $25 \mathrm{~kg} / \mathrm{m}^{2}$, surpoids : 25 à $29 \mathrm{~kg} / \mathrm{m}^{2}$, obésité : $30 \mathrm{~kg} / \mathrm{m}^{2}$ ou plus), en fonction de la consommation d'alcool (fréquence hebdomadaire, fréquence moins qu'hebdomadaire, aucune consommation au cours des 12 derniers mois), en fonction du tabagisme (sujet n'ayant jamais fumé, ancien fumeur, fumeur), en fonction de la consommation de fruits et légumes (moins de 5 portions par jour ou 5 et plus) et en fonction du degré d'activité physique (sujet actif, modérément actif, sédentaire). Le degré d'activité physique est fondé sur une estimation de la dépense énergétique quotidienne totale, laquelle est calculée à partir de la fréquence et de la durée autodéclarées de l'activité physique pratiquée durant les loisirs ou pour le transport au cours des trois mois précédant l'enquête ${ }^{15}$.

Nous avons également examiné la présence concomitante d'autres affections chroniques. Nous avons défini la comorbidité comme étant le nombre d'autres affections chroniques déclarées, et nous avons réparti les sujets en deux groupes : ceux ayant déclaré moins de trois affections comorbides et ceux en ayant déclaré trois ou plus. Ce seuil a été déterminé en fonction des résultats de notre analyse bivariée, selon lesquels l'une des caractéristiques du SFC comme de la FM est que la quasi-totalité des répondants atteints de ces affections présente également au moins une ou deux autres affections chroniques. Les affections chroniques mentionnées dans l'ESCC de 2010 étaient l'asthme, l'arthrite, les maux de dos, la maladie pulmonaire obstructive chronique (MPOC), les troubles intestinaux, les sensibilités aux agresseurs chimiques, les migraines, l'hypertension, les maladies cardiaques, le diabète, le cancer, les ulcères à l'estomac, l'incontinence urinaire, les troubles de l'humeur, les troubles d'anxiété, la maladie d'Alzheimer ou une autre forme de démence, la sclérose latérale amyotrophique, la paralysie cérébrale, la dystonie, l'épilepsie, l'hydrocéphalie, la maladie d'Huntington, la dystrophie musculaire, la sclérose en plaques, la maladie de Parkinson, le spina bifida, les accidents vasculaires cérébraux, le syndrome de Tourette et les problèmes neurologiques causés par une blessure au cerveau, une blessure à la moelle épinière ou une tumeur.

\section{Indicateurs de l'état de santé}

Six indicateurs autodéclarés de l'état de santé ont été mesurés chez les Canadiens atteints à la fois du SFC et de FM, chez ceux atteints uniquement du SFC, chez ceux atteints uniquement de FM et chez ceux atteints ni du SFC ni de la FM : santé générale passable ou mauvaise, santé mentale passable ou mauvaise, limitation des activités, aide requise pour certaines tâches, incapacité sévère et, enfin, douleur.

- Santé générale et santé mentale passables ou mauvaises. Nous avons défini l'état de santé générale et l'état de santé mentale en fonction des réponses aux questions suivantes : « En général, diriez-vous que votre santé est : excellente, très bonne, bonne, passable ou mauvaise? » et « En général, diriez-vous que votre santé mentale est : excellente, très bonne, bonne, passable ou mauvaise? ». Nous avons classé les réponses à ces questions en deux groupes, soit santé passable ou mauvaise d'une part et santé excellente, très bonne ou bonne d'autre part.

- Limitation des activités. Nous avons établi une mesure de la limitation des activités quotidiennes à partir des réponses fournies par les répondants (« souvent », " parfois », " jamais ») aux cinq questions suivantes : 1) " Avezvous de la difficulté à entendre, à voir, à communiquer, à marcher, à monter un escalier, à vous pencher, à apprendre ou à faire d'autres activités semblables? » et « Est-ce qu'un état physique ou un état mental ou un problème de santé de longue durée réduit la quantité ou le genre d'activités que vous pouvez faire : 2) à la maison? 3) à l'école? 4) au travail? 5) dans d'autres contextes, par exemple dans les déplacements ou les loisirs? " Nous avons considéré que les répondants avaient des limitations pour certaines activités s'ils avaient répondu « souvent » ou "parfois » à au moins une de ces cinq questions.
- Aide requise pour certaines tâches. Nous avons considéré que les répondants avaient besoin d'aide pour certaines tâches s'ils avaient déclaré avoir besoin de l'aide d'une autre personne pour effectuer au moins l'une des six activités quotidiennes suivantes : préparer les repas, se rendre à un rendez-vous ou faire des commissions, accomplir des tâches ménagères, assurer ses soins personnels, se déplacer dans la maison, s'occuper de ses finances personnelles.

- Incapacité sévère. Nous avons mesuré la qualité de vie liée à la santé à l'aide de l'Indice de l'état de santé (HUI - Health Utilities Index). Il est formé de 8 attributs (vision, ouie, parole, ambulation, dextérité, émotions, cognition, douleurs et malaises) et comporte de cinq à six niveaux fonctionnels par attribut. Nous utilisons une fonction d'utilité pour obtenir un score global de l'état de santé allant de $-0,36$ à 1,0 (où $-0,36$ indique un état de santé pire que la mort, 0,0 un état de santé équivalant à la mort et 1,0 une santé parfaite). Nous avons regroupé les scores de l'état de santé en deux catégories selon le degré d’incapacité : incapacité nulle à modérée (de 0,70 à 1,00 ) et incapacité sévère (moins de $0,70)$.

- La présence d'une douleur a été déterminée à l'aide de la question suivante : «Êtes-vous habituellement sans douleur ou malaise? » (« oui » ou « non »).

\section{Analyse statistique}

Nous avons analysé les données au moyen du logiciel SAS Enterprise Guide, version 5.1 (SAS Institute Inc., Cary [Caroline du Nord], États-Unis). Le degré de signification statistique a été fixé à une valeur $p$ inférieure à 0,05 dans toutes les analyses. Afin de tenir compte de la répartition de l'échantillon et de la conception de l'enquête, nous avons pondéré toutes les estimations en fonction de la pondération utilisée par Statistique Canada et nous avons estimé les intervalles de confiance (IC) à $95 \%$ en utilisant la méthode de rééchantillonnage bootstrap. Nous avons quantifié les associations en utilisant les rapports de prévalence estimés par régres- 
sion binomiale multivariée, avec une valeur à l'origine de -4 pour améliorer la convergence $^{16}$.

\section{Résultats}

\section{Prévalence du SFC et de la FM}

En 2010, environ 411000 (1,4 \%; IC à $95 \%$ : $1,3 \%$ à 1,6 \%) Canadiens de 12 ans ou plus avaient déclaré avoir reçu un diagnostic de SFC, et 444000 (1,5\%; IC à $95 \%: 1,4 \%$ à $1,7 \%$ ) un diagnostic de FM. Environ 0,3 \% (IC à $95 \%: 0,3 \%$ à 0,4\%) de la population totale vivant à domicile avait déclaré souffrir de ces deux affections. Environ une personne sur quatre parmi celles atteintes du SFC $(23,0 \%)$ avait déclaré souffrir également de FM, et une personne sur cinq parmi celles atteintes de FM (21,2\%) avait déclaré souffrir également du SFC. Dans l'ensemble, la prévalence du SFC comme de la FM était plus élevée chez les femmes dans tous les groupes d'âge (figure 1).

\section{Facteurs associés à la prévalence du SFC et de la FM}

Après ajustement en fonction des covariables, les femmes, les adultes de 40 ans ou plus et les personnes à plus faible revenu étaient plus susceptibles de déclarer avoir reçu un diagnostic de SFC ou de FM (tableau 1). De plus, dans les analyses multivariées, le SFC était associé à la sédentarité, au fait de fumer ou d'avoir fumé et à une consommation peu fréquente d'alcool. La FM était associée à l'obésité et à une consommation d'alcool nulle ou moins qu'hebdomadaire. Les affections comorbides étaient fréquentes chez les personnes atteintes du SFC comme de FM, 65,2 \% (IC à $95 \%$ : $59,9 \%$ à 70,6 \%) de ces personnes ayant déclaré être atteintes d'au moins trois autres affections concomitantes.

\section{Répercussions du SFC ou de la FM sur l'état de santé}

Les Canadiens atteints du SFC ou de FM répondaient aux critères des indicateurs d'un mauvais état de santé plus souvent que les Canadiens ne souffrant ni du SFC ni de FM (tableau 2). Après ajustement en fonction des différences quant au nombre d'affections chroniques concomitantes, des données sociodémographiques et des facteurs de risque pour la santé, les personnes atteintes du SFC ou de FM

FIGURE 1

Prévalence du syndrome de fatigue chronique, de la fibromyalgie et de la présence concomitante de ces deux maladies en fonction de l'âge et du sexe chez les Canadiens de 12 ans ou plus, Enquête sur la santé dans les collectivités canadiennes de 2010

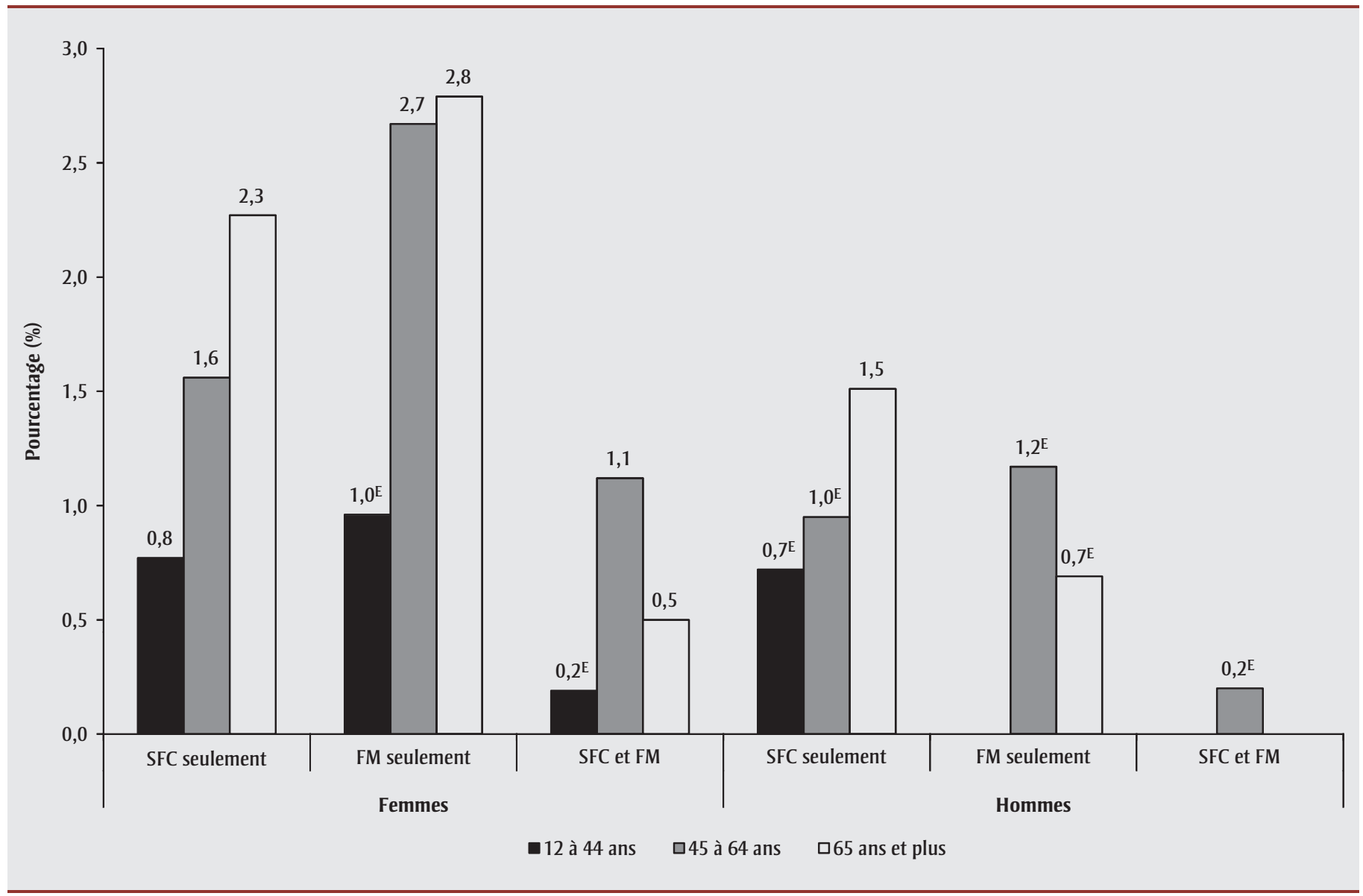

Abréviations : FM, fibromyalgie; SFC, syndrome de fatigue chronique.

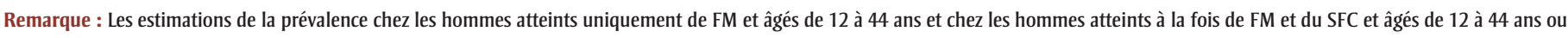
de 65 ans ou plus ne sont pas présentées, car la variabilité de l'échantillonnage était élevée.

${ }^{\mathrm{E}}$ Interpréter avec prudence, car le coefficient de variation est situé entre 16,6 \% et 33,3\%. 
TABLEAU 1

Prévalence du syndrome de fatigue chronique et de la fibromyalgie en fonction d'une série de caractéristiques sociodémographiques et de caractéristiques liées à la santé, population de 12 ans ou plus, Enquête sur la santé dans les collectivités canadiennes de 2010

\begin{tabular}{|c|c|c|c|c|c|c|}
\hline \multirow[t]{2}{*}{ Caractéristiques } & \multicolumn{3}{|r|}{ SFC } & \multicolumn{3}{|r|}{ FM } \\
\hline & $\mathbf{N}$ & $\%$ & $\begin{array}{l}\text { Analyse multivariée } \\
\text { Rapports de préva- } \\
\text { lence (IC à } 95 \% \text { ) }\end{array}$ & $\mathbf{N}$ & $\%$ & $\begin{array}{l}\text { Analyse multivariée } \\
\text { Rapports de préva- } \\
\text { lence (IC à } 95 \% \text { ) }\end{array}$ \\
\hline \multicolumn{7}{|l|}{ Sexe } \\
\hline Masculin & 313 & 1,0 & Pt de réf. & 157 & $0,7^{\mathrm{E}}$ & Pt de réf. \\
\hline Féminin & 693 & 1,8 & $1,7(1,2-2,2)$ & 956 & 2,4 & $3,5(2,3-5,4)$ \\
\hline \multicolumn{7}{|l|}{ Âge (ans) } \\
\hline 12 à 39 & 160 & 0,8 & Pt de réf. & 103 & $0,4^{\mathrm{E}}$ & Pt de réf. \\
\hline 40 à 59 & 378 & 1,8 & $2,1(1,3-3,4)$ & 472 & 2,3 & $4,3(2,7-6,9)$ \\
\hline 60 et plus & 468 & 2,2 & $2,0(1,3-3,2)$ & 538 & 2,6 & $3,5(2,2-5,8)$ \\
\hline \multicolumn{7}{|l|}{ Origine ethnique } \\
\hline Blanc & 861 & 1,5 & Pt de réf. & 996 & 1,6 & Pt de réf. \\
\hline Autochtone hors réserve & 66 & $2,3^{\mathrm{E}}$ & $1,5(0,9-2,4)$ & 54 & $1,7^{\mathrm{E}}$ & $1,2(0,7-1,9)$ \\
\hline Autre & $\geq 60$ & $1,2^{\mathrm{E}}$ & $0,9(0,5-1,5)$ & 47 & $1,2^{\mathrm{E}}$ & $0,6(0,3-1,5)$ \\
\hline \multicolumn{7}{|l|}{ Niveau de scolarité } \\
\hline Diplôme d'études post-secondaires & 440 & 1,3 & Pt de réf. & 562 & 1,5 & Pt de réf. \\
\hline Études post-secondaires partielles & 76 & $1,2^{\mathrm{E}}$ & $1,4(0,9-2,2)$ & 73 & $1,5^{\mathrm{E}}$ & $1,0(0,6-1,6)$ \\
\hline Diplôme d'études secondaires & 180 & 1,7 & $0,9(0,6-1,3)$ & 177 & 1,6 & $0,8(0,5-1,1)$ \\
\hline Études secondaires non terminées & 287 & 1,8 & $1,1(0,8-1,5)$ & 281 & 1,6 & $0,8(0,6-1,1)$ \\
\hline Non précisé & 57 & $1,5^{\mathrm{E}}$ & $1,4(0,8-2,5)$ & 48 & $1,5^{\mathrm{E}}$ & $1,2(0,5-3,2)$ \\
\hline \multicolumn{7}{|l|}{ Suffisance du revenu } \\
\hline $5^{\mathrm{e}}$ quintile (le plus élevé) & 94 & $0,8^{\mathrm{E}}$ & Pt de réf. & 139 & 1,0 & Pt de réf. \\
\hline $4^{\mathrm{e}}$ quintile & 126 & 0,9 & $1,1(0,7-1,8)$ & 172 & 1,7 & $1,7(1,1-2,6)$ \\
\hline $3^{\mathrm{e}}$ quintile & 148 & 1,3 & $1,5(0,8-2,5)$ & 190 & 1,4 & $1,2(0,7-1,9)$ \\
\hline $2^{\mathrm{e}}$ quintile & 245 & 1,6 & $1,6(1,0-2,7)$ & 252 & 1,4 & $1,1(0,8-1,7)$ \\
\hline $1^{\text {er }}$ quintile (le plus faible) & 379 & 2,5 & $2,3(1,4-3,9)$ & 347 & 2,1 & $1,6(1,0-2,4)$ \\
\hline Non précisé & - & $\mathrm{F}$ & & - & $\mathrm{F}$ & \\
\hline \multicolumn{7}{|l|}{ État matrimonial } \\
\hline Célibataire & 191 & 1,0 & Pt de réf. & 137 & 0,6 & Pt de réf. \\
\hline Marié(e)/conjoint(e) de fait & 462 & 1,4 & $1,1(0,7-1,8)$ & 402 & 3,8 & $1,6(0,9-2,8)$ \\
\hline Veuf(ve)/séparé(e)/divorcé(e) & 348 & 2,7 & $1,0(0,6-1,4)$ & 571 & 1,5 & $1,2(0,7-1,8)$ \\
\hline \multicolumn{7}{|l|}{ Indice de masse corporelle, $\mathrm{kg} / \mathrm{m}^{2}$} \\
\hline Moins de 25 & 375 & 1,1 & Pt de réf. & 371 & 1,2 & Pt de réf. \\
\hline 25 à 29 & 281 & 1,4 & $1,2(0,9-1,6)$ & 356 & 1,6 & $1,3(0,9-1,7)$ \\
\hline 30 et plus & 254 & 1,8 & $1,2(0,9-1,6)$ & 319 & 2,3 & $1,5(1,1-2,1)$ \\
\hline \multicolumn{7}{|l|}{ Degré d'activité physique } \\
\hline Sujets actifs & 151 & 0,8 & Pt de réf. & 170 & 1,0 & Pt de réf. \\
\hline Sujets modérément actifs & 170 & 1,1 & $1,2(0,8-1,9)$ & 234 & 1,1 & $0,8(0,6-1,3)$ \\
\hline Sujets sédentaires & 624 & 1,8 & $1,6(1,2-2,2)$ & 688 & 2,0 & $1,4(1,0-1,8)$ \\
\hline \multicolumn{7}{|l|}{ Consommation d'alcool } \\
\hline Au moins hebdomadaire & 237 & 0,9 & Pt de réf. & 296 & 1,2 & Pt de réf. \\
\hline Moins qu'hebdomadaire & 419 & 1,7 & $1,5(1,2-2,0)$ & 435 & 1,6 & $1,3(1,0-1,8)$ \\
\hline Pas au cours des 12 derniers mois & 336 & 2,0 & $1,8(1,3-2,6)$ & 369 & 2,1 & $1,8(1,3-2,5)$ \\
\hline \multicolumn{7}{|l|}{ Tabagisme } \\
\hline Jamais fumé & 272 & 1,0 & Pt de réf. & 333 & 1,3 & Pt de réf. \\
\hline
\end{tabular}

Suite page suivante étaient entre 1,2 et 1,9 fois plus susceptibles de déclarer un mauvais état de santé (en fonction de cinq des indicateurs) que celles atteintes d'aucune de ces affections (tableau 3). Nous n'avons constaté aucune différence statistiquement significative pour ce qui est du sixième indicateur, à savoir une santé mentale autodéclarée passable ou mauvaise.

Facteurs associés à un mauvais état de santé chez les personnes atteintes du SFC ou de FM

Les facteurs le plus souvent associés aux indicateurs d'un mauvais état de santé chez les personnes atteintes du SFC ou de FM étaient le fait d'avoir reçu un diagnostic à la fois de SFC et de FM, le fait d'avoir reçu un diagnostic d'au moins trois autres affections chroniques et le fait d'être sédentaire (tableau 4), indépendamment des données sociodémographiques et des caractéristiques liées à la santé. Par rapport aux personnes ne souffrant ni du SFC ni de FM, les sujets atteints des deux affections étaient entre 1,3 et 1,6 fois plus susceptibles de déclarer avoir une santé générale passable ou mauvaise, d'avoir une incapacité sévère (d'après l'indice de l'état de santé), de ressentir de la douleur, d'avoir des limitations pour certaines activités et d'avoir besoin d'aide pour effectuer des tâches quotidiennes. De plus, les personnes atteintes du SFC ou de FM ainsi que de trois autres affections chroniques ou plus étaient entre 1,6 et 2,9 fois plus susceptibles de déclarer ces indicateurs de mauvaise santé. Enfin, les personnes considérées comme sédentaires étaient de 1,2 à 1,8 fois plus susceptibles de déclarer une santé générale passable ou mauvaise, une incapacité sévère, des limitations dans certaines activités et un besoin d'aide pour effectuer certaines tâches. En outre, certains facteurs sociodémographiques et liés au mode de vie étaient associés à un ou à deux indicateurs de mauvaise santé (tableau 4).

\section{Analyse}

Nous avons utilisé les données d'une enquête représentative de la population à l'échelle nationale pour estimer la prévalence et les corrélats du SFC et de la 
TABLEAU 1 (Suite)

Prévalence du syndrome de fatigue chronique et de la fibromyalgie en fonction d'une série de caractéristiques sociodémographiques et de caractéristiques liées à la santé, population de 12 ans ou plus, Enquête sur la santé dans les collectivités canadiennes de 2010

\begin{tabular}{|c|c|c|c|c|c|c|}
\hline \multirow[t]{2}{*}{ Caractéristiques } & \multicolumn{3}{|r|}{ SFC } & \multicolumn{3}{|r|}{ FM } \\
\hline & $\mathbf{N}$ & $\%$ & $\begin{array}{l}\text { Analyse multivariée } \\
\text { Rapports de préva- } \\
\text { lence (IC à } 95 \% \text { ) }\end{array}$ & $\mathbf{N}$ & $\%$ & $\begin{array}{l}\text { Analyse multivariée } \\
\text { Rapports de préva- } \\
\text { lence (IC à } 95 \% \text { ) }\end{array}$ \\
\hline Ancien fumeur & 392 & 1,4 & $1,7(1,2-2,4)$ & 499 & 1,8 & $1,2(0,8-1,8)$ \\
\hline Fumeur & 336 & 2,3 & $2,7(1,9-3,8)$ & 276 & 1,6 & $1,3(0,8-1,9)$ \\
\hline \multicolumn{7}{|l|}{ Consommation de fruits et légumes } \\
\hline Moins de 5 portions par jour & 549 & 1,3 & Pt de réf. & 572 & 1,6 & Référent \\
\hline 5 portions ou plus par jour & 336 & 1,3 & $1,2(0,9-1,6)$ & 467 & 1,4 & $0,9(0,7-1,1)$ \\
\hline
\end{tabular}

Abréviations : FM, fibromyalgie; IC, intervalle de confiance; Pt de réf., point de référence; SFC, syndrome de fatigue chronique.

Remarque : Les associations statistiquement significatives $(p<0,05)$ sont présentées en caractères gras.

${ }^{E}$ Interpréter avec prudence (le coefficient de variation est situé entre 16,6 \% et 33,3\%).

F Donnée trop incertaine pour être déclarée (coefficient de variation supérieur à 33,3\%).

FM : en 2010, environ $1,4 \%$ de la population canadienne vivant à domicile indiquait avoir reçu un diagnostic de SFC et $1,5 \%$ un diagnostic de FM, soit respectivement 411000 et 444000 Canadiens de 12 ans ou plus.

Tout comme l'ont révélé d'autres données recueillies au Canada et à l'étranger ${ }^{1,14,17}$, nous avons constaté qu'il y avait un lien entre le fait d'être une femme, celui d'avoir 40 ans ou plus et celui d'avoir un faible revenu et le fait d'être atteint du SFC ou de FM. Étant donné la nature transversale de l'enquête, il demeure difficile de déterminer si le faible statut socioéconomique des personnes atteintes de ces affections est un déterminant ou une conséquence du SFC et de la FM. En effet, le SFC et la FM peuvent avoir une incidence sur la capacité d'une personne à travailler et, par conséquent, sur le revenu total du ménage. Dans une étude menée au Royaume-Uni auprès de personnes atteintes du SFC, Collin et collab. ${ }^{18}$ ont constaté que $50 \%$ d'entre elles cessaient de travailler en raison des symptômes liés au SFC. Selon les auteurs, la perte de productivité associée au SFC coûte de $75 \mathrm{M} £$ à $129 \mathrm{M} £$ à l'économie britannique ${ }^{18}$. De façon analogue, Reynolds et collab. ${ }^{19}$ ont estimé que le SFC entraînait une baisse de productivité de $37 \%$ au sein d'un ménage et une baisse de productivité de la main-d'œuvre de l'ordre de 54 \% . La valeur annuelle totale de la perte de productivité aux États-Unis était d'environ 9,1 G\$, soit
20000 \$ par personne atteinte du SFC. Selon Knight et collab. ${ }^{20}$, la perte de productivité due à une incapacité causée par la FM coûterait à l'économie américaine 7333 \$ par patient, et la perte de productivité due à l'absentéisme coûterait 1228 \$ par patient. Comme l'incapacité à travailler ou la diminution des heures travaillées en raison du SFC ou de la FM peut avoir une incidence sur le revenu, il est possible qu'un faible revenu ne soit pas un déterminant mais plutôt une conséquence de ces affections.

Nous avons également montré, ce qui est conforme aux résultats de l'ESCC de 2000$2001^{14}$, qu'il existe une relation entre les facteurs de risque de maladie chronique liés au mode de vie (obésité, sédentarité, tabagisme) et le SFC ou la FM. Il convient toutefois de souligner que la direction de cette relation demeure incertaine, étant donné la nature transversale des données. D'après notre analyse, les personnes obèses étaient 1,5 fois plus susceptibles de déclarer être atteintes de FM. Ursini et collab. ${ }^{21}$ ont émis l'hypothèse que la FM et l'obésité étaient liées par un certain nombre de mécanismes, notamment la sédentarité, les troubles du sommeil, la dépression, un dysfonctionnement de la thyroïde et des perturbations hormonales liées à la dérégulation du facteur de croissance insulinomimétique.

Dans notre analyse, la sédentarité autodéclarée est liée au fait d'avoir reçu un

TABLEAU 2

Résultats en matière de santé des Canadiens de 12 ans ou plus ayant déclaré être atteints d'un syndrome de fatigue chronique ou de fibromyalgie diagnostiqués par un professionnel de la santé, Enquête sur la santé dans les collectivités canadiennes de 2010

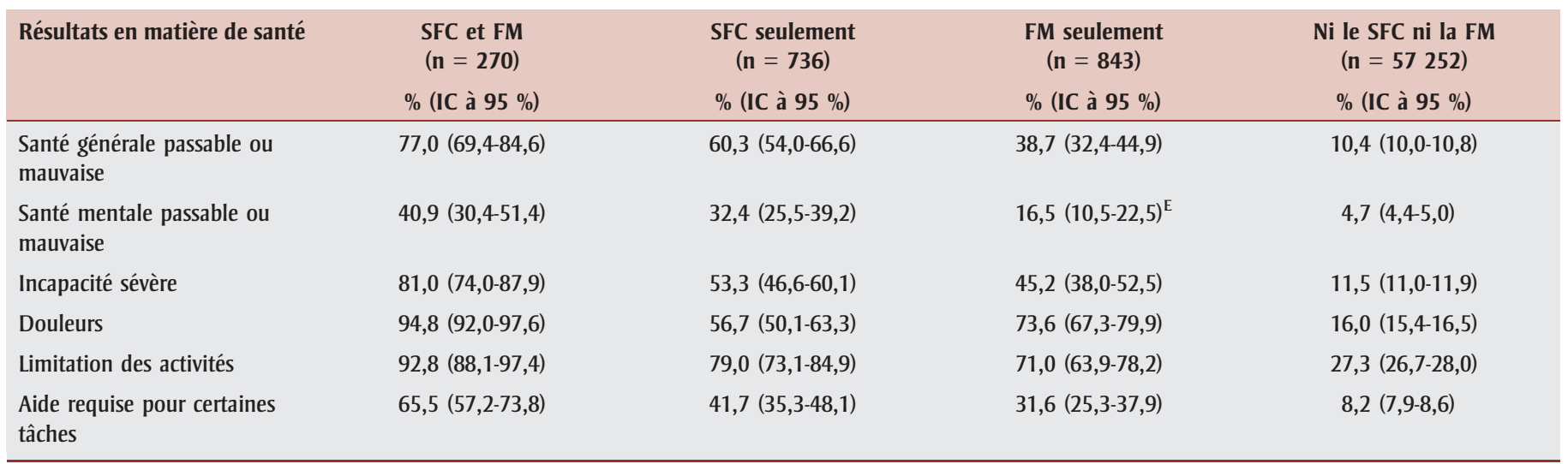

Abréviations : FM, fibromyalgie; IC, intervalle de confiance; SFC, syndrome de fatigue chronique.

E Interpréter avec prudence (le coefficient de variation est situé entre 16,6 \% et 33,3\%). 
TABLEAU 3

Associations entre le syndrome de fatigue chronique ainsi que la fibromyalgie et divers indicateurs de l'état de santé chez les Canadiens de 12 ans ou plus, Enquête sur la santé dans les collectivités canadiennes de 2010

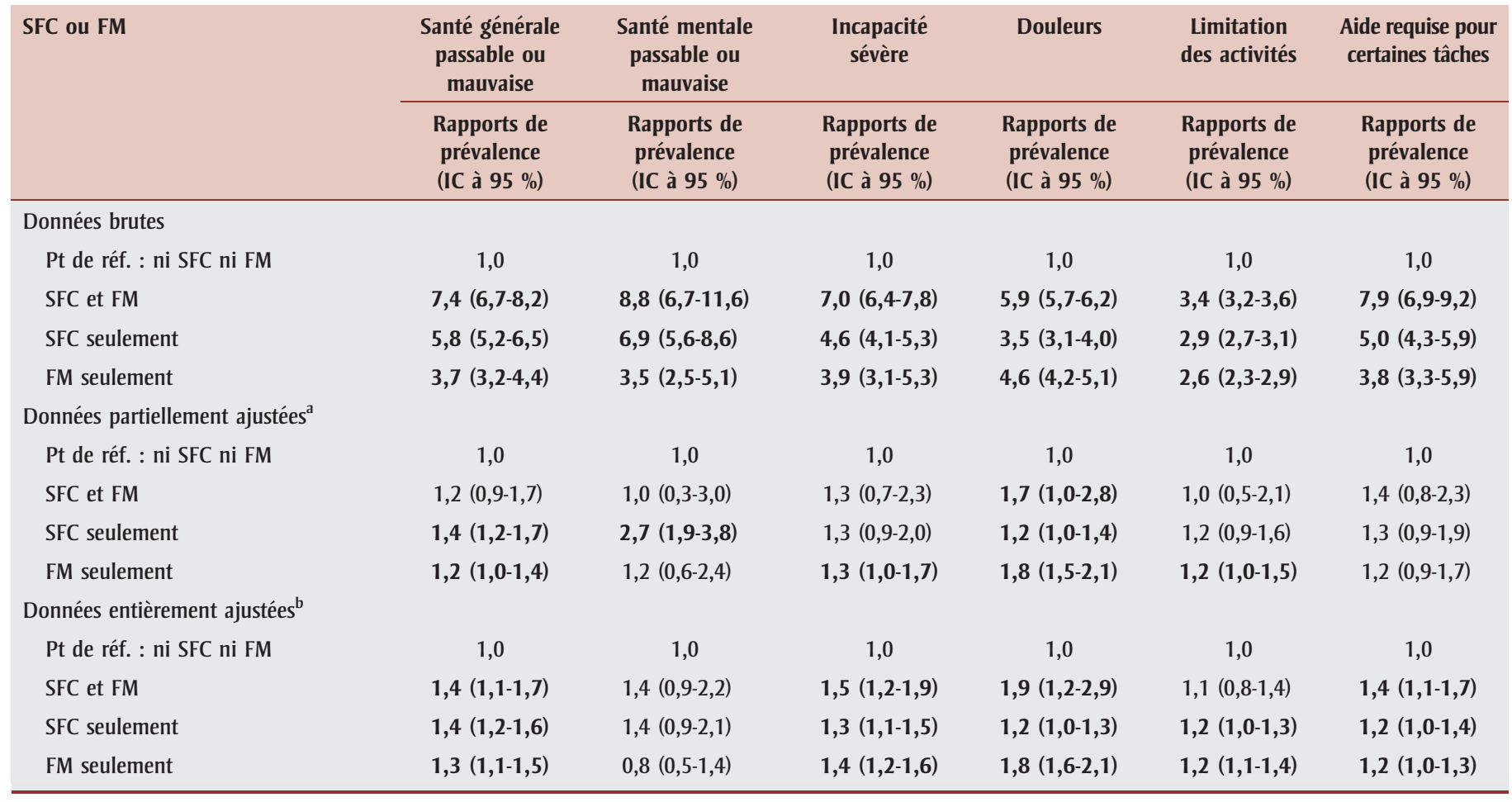

Abréviations : FM, fibromyalgie; IC, intervalle de confiance; Pt de réf., point de référence; SFC, syndrome de fatigue chronique.

Remarque : Les associations statistiquement significatives $(p<0,05)$ sont présentées en caractères gras.

a Ajusté en fonction du nombre d'affections chroniques comorbides (en continu).

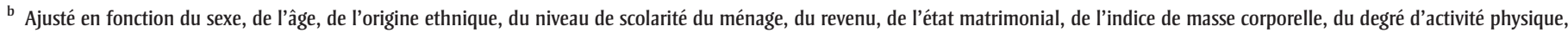
de la consommation d'alcool, du tabagisme, de la consommation de fruits et légumes et du nombre d'affections chroniques comorbides (en continu).

diagnostic de SFC. En utilisant les données de l'étude prospective National Child Development Study, menée auprès d'une cohorte de personnes nées en 1958 en Angleterre, au pays de Galles et en Écosse, Goodwin et collab. ${ }^{22}$ ont montré qu'il n'y avait aucune relation entre la pratique hebdomadaire d'une activité physique à 23 ans et à 33 ans et l'apparition d'un SFC avant l'âge de 42 ans. Cette absence de corrélation est en opposition avec les résultats de la cohorte de personnes nées en 1946 dans ces mêmes régions, qui montrent que la pratique d'activité physique à une fréquence accrue pendant l'enfance et au début de la vie adulte était un prédicteur du SFC avant l'âge de 53 ans $^{23}$. Bien que, à notre connaissance, cette relation n'ait été examinée que dans le cadre de ces deux études prospectives, ces résultats donnent à penser que la sédentarité est plus vraisemblablement une conséquence du SFC qu'une cause. La sédentarité peut découler de la fatigue, de la douleur et des incapacités physiques accrues observables dans les cas de SFC et de FM. Dans notre analyse, la sédentarité était liée à ces facteurs.

Notre étude a révélé qu'il y avait une relation entre le fait de fumer ou d'avoir fumé et le SFC. À notre connaissance, personne n'a cherché dans le cadre d'une étude prospective à savoir si le tabagisme était un facteur de risque du SFC.

La question des affections comorbides, que ce soit le fait de souffrir à la fois du SFC et de FM ou d'être atteint d'une autre affection chronique en plus du SFC ou de la FM, est centrale dans le cas de la population étudiée ici. D’autres études ont révélé que, chez les patients ayant reçu à la fois un diagnostic de FM et un diagnostic de SFC, l'évolution de ces maladies était plus défavorable, l'état de santé général et le degré de satisfaction par rapport à cet état de santé étaient moindres et les répercussions des maladies étaient plus importantes que chez les personnes atteintes uniquement du SFC ou de $\mathrm{FM}^{2,24}$. Nos résultats montrent également que le degré de comorbidité d'une personne peut avoir une incidence considérable sur les résultats liés à son état de santé. De plus, les deux tiers des personnes atteintes du SFC ou de FM ont déclaré souffrir d'au moins trois autres affections chroniques. Notre analyse a révélé que le nombre d'affections concomitantes chez les personnes atteintes du SFC ou de FM expliquait en grande partie les différences observées quant à l'état de santé par rapport aux personnes ne souffrant d'aucune de ces deux affections. Ainsi, nos résultats révèlent qu'il est important de tenir compte de l'effet cumulatif des affections chroniques concomitantes et du SFC et de la FM lorsqu'on examine les effets sur la santé chez des personnes atteintes de l'une ou l'autre de ces maladies ou des deux. 
TABLEAU 4

Associations ajustées en fonction des analyses multivariées entre les caractéristiques et les indicateurs de l'état de santé des Canadiens de 12 ans ou plus atteints du syndrome de fatigue chronique ou de fibromyalgie ( $n=1849$ ), Enquête sur la santé dans les collectivités canadiennes de 2010

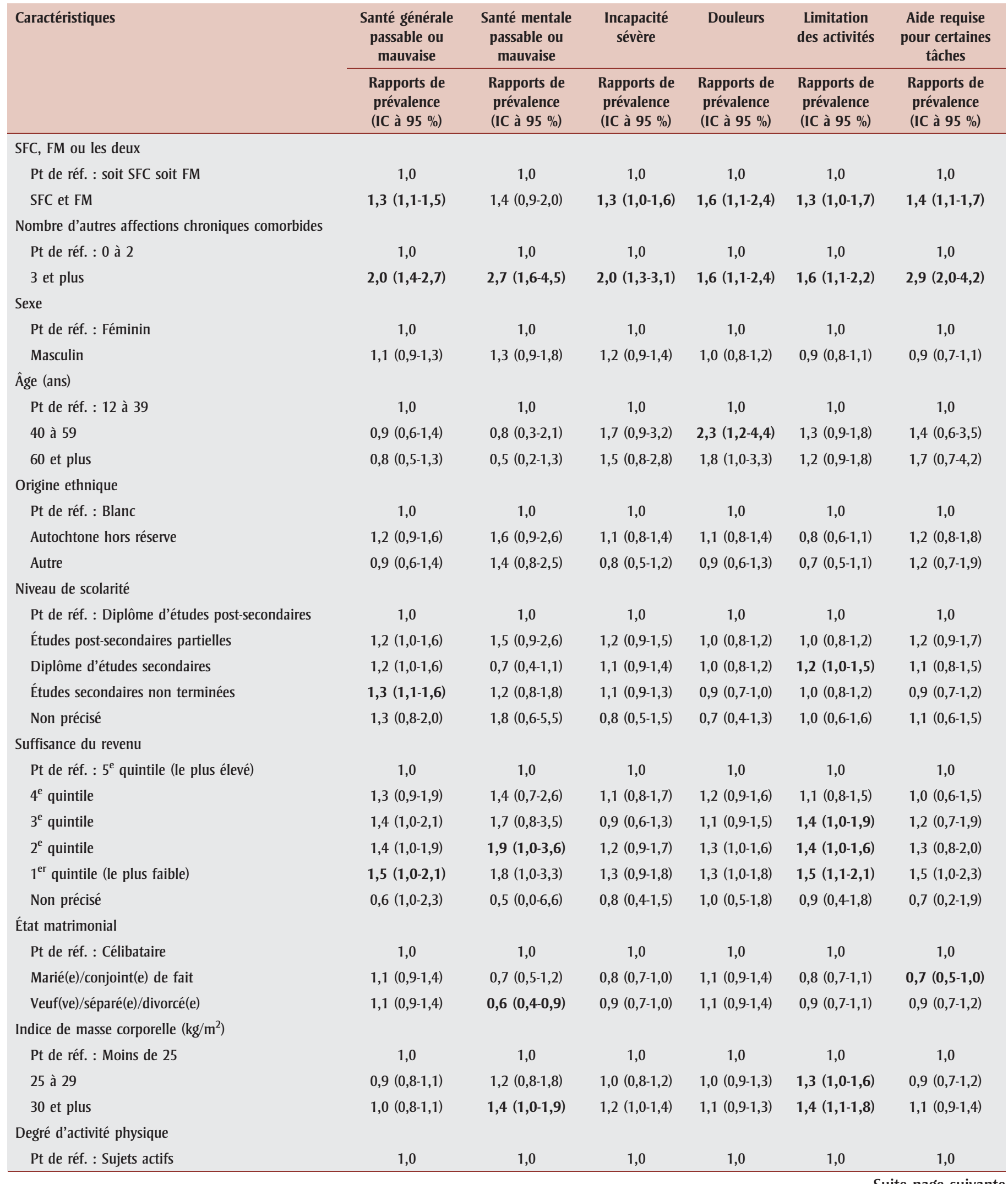


TABLEAU 4 (Suite)

Associations ajustées en fonction des analyses multivariées entre les caractéristiques et les indicateurs de l'état de santé des Canadiens de 12 ans ou plus atteints du syndrome de fatigue chronique ou de fibromyalgie $(n=1849)$, Enquête sur la santé dans les collectivités canadiennes de 2010

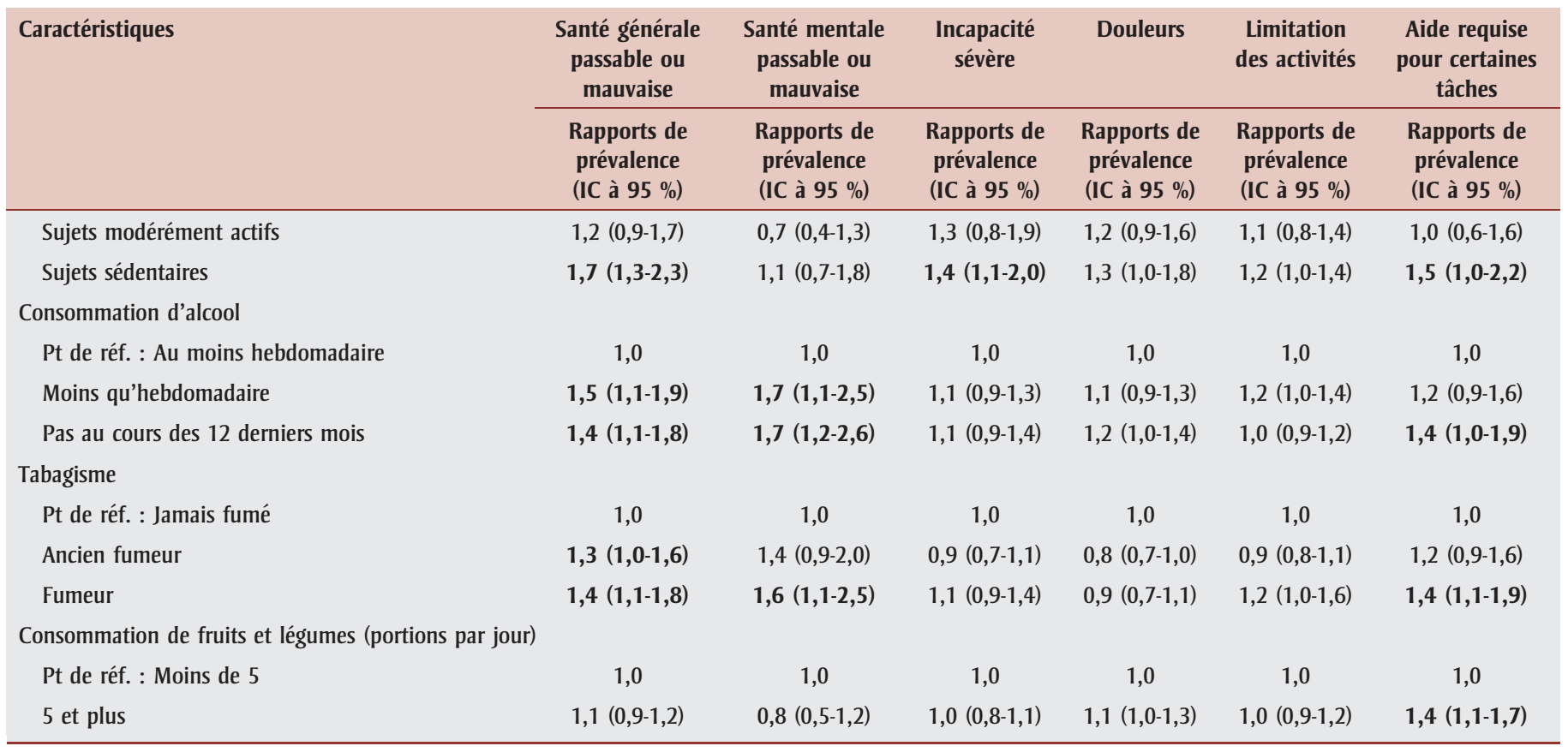

Abréviations : FM, fibromyalgie; Pt de réf., point de référence; SFC, syndrome de fatigue chronique.

Remarque : Les associations statistiquement significatives $(p<0,05)$ sont présentées en caractères gras.

\section{Points forts et limites}

L'un des points forts de notre étude est qu'elle est fondée sur une enquête de grande envergure menée auprès de la population canadienne vivant dans la collectivité et ayant obtenu un bon taux de réponse. L'ESCC fournit des données exhaustives sur plusieurs variables descriptives, ce qui permet d'analyser de façon approfondie l'état de santé des personnes atteintes du SFC ou de FM et de comparer ces personnes avec divers sous-groupes. L'ESCC est fondée sur l'autodéclaration d'affections chroniques et d'événements touchant la santé. Bien qu'il s'agisse du moyen le plus pratique d'évaluer des états pathologiques dans des études menées auprès de populations de taille importante, l'autodéclaration des diagnostics est sujette à un risque de classification erronée, ce qui peut entraîner une surestimation ou une sous-estimation de la prévalence d'une maladie et du fardeau sociétal de celle-ci. Dans notre étude, les répondants de l'ESCC avaient déclaré eux-mêmes leurs antécédents médicaux (notamment les diagnostics de SFC ou de FM), et aucune vérification de l'information autodéclarée n'avait été effec- tuée par un tiers. Des chercheurs ont constaté qu'il y avait une correspondance d'acceptable à bonne entre les problèmes de santé physique autodéclarés et les diagnostics posés par les médecins ${ }^{25}$, mais il n'y a eu aucune validation, à notre connaissance, des renseignements autodéclarés concernant expressément le SFC et la FM. Les études portant sur les pratiques diagnostiques et axées sur les définitions de cas utilisées par les professionnels de la santé pour poser un diagnostic de SFC ou de FM sont rares, et aucune n'a pour l'instant été réalisée au Canada.

Comme nous l'avons mentionné précédemment, le caractère transversal de l'enquête ne nous permet pas d'examiner les mécanismes ou les liens de causalité possibles, ce qui fait qu'il est difficile de savoir si les associations que nous avons observées concernant les comportements à risque associés au mode de vie doivent être considérées comme relevant des facteurs de risque de ces affections ou des résultats découlant de ces affections. Il faudrait mener des études étiologiques (études cas-témoins ou études de cohorte par exemple) pour déterminer si, dans le cas du SFC et de la FM, il s'agit de facteurs de risque potentiellement évitables. Enfin, bien que nous ayons tenu compte, dans notre stratégie analytique, de toutes les covariables importantes mentionnées dans les publications sur le SFC et la FM, nos analyses se sont limitées aux variables mesurées par l'ESCC. Cela peut donc avoir entraîné l'exclusion de covariables importantes comme la gravité ou la durée de la maladie, susceptibles de constituer des facteurs de confusion sur la nature des associations révélées dans l'étude.

\section{Conclusion}

Nous avons observé que, en 2010, la population canadienne vivant à domicile âgée de 12 ans ou plus avait déclaré être atteinte du SFC dans une proportion de 1,4 \% et de FM dans une proportion de 1,5\%. Nous avons également observé que les cas de SFC et de FM étaient associés au fait d'être une femme, aux adultes de 40 ans ou plus et à certains facteurs de risque de maladies chroniques associés au mode de vie, mais les raisons à l'origine de ces associations demeurent nébuleuses. À la lumière de ces observations, il serait 
judicieux d'effectuer d'autres travaux de recherche pour déterminer si ces facteurs de risque associés au mode de vie sont une cause ou un effet du SFC et de la FM. Le fait d'avoir une moins bonne santé était étroitement corrélé à la présence concomitante du SFC, de la FM et d'autres affections chroniques. On ne saurait donc faire abstraction du rôle déterminant joué par les affections comorbides dans la dégradation de l'état de santé.

Étant donné le manque relatif de données sur le SFC et la FM, ces résultats issus d'une enquête menée dans la collectivité sont pertinents. Ils renforcent les observations antérieures voulant que ces maladies s'accompagnent fréquemment d'une série d'affections. Étant donné qu'il est rare que l'on soit atteint du SFC ou de FM sans souffrir d'une affection comorbide, les chercheurs et les cliniciens peuvent s'attendre à ce que les études à mener et les soins à prodiguer soient très complexes. Il serait particulièrement pertinent, pour les praticiens œuvrant dans le domaine de la santé publique et pour les autres professionnels de la santé, de ne pas exclure les patients atteints d'affections comorbides des futures recherches. Enfin, la compréhension des facteurs contribuant à l'amélioration de la qualité de vie des personnes atteintes du SFC ou de FM, et en particulier des personnes qui souffrent de ces deux maladies ou d'affections comorbides, constituerait sans doute un domaine de recherche important pour de futurs travaux.

\section{Remerciements}

L'Enquête sur la santé dans les collectivités canadiennes a été menée par Statistique Canada en partenariat avec Santé Canada et l'Agence de la santé publique du Canada, grâce à l'appui financier du gouvernement fédéral du Canada.

\section{Références}

1. Park J, Knudson S. Symptômes physiques médicalement inexpliqués. Rapports sur la santé. 2007;18:49-54.
2. Lavergne MR, Cole DC, Kerr K, Marshall LM. Functional impairment in chronic fatigue syndrome, fibromyalgia, and multiple chemical sensitivity. Can Fam Physician. 2010; 56:e57-e65.

3. Jason LA, Taylor RR, Kennedy CL. Chronic fatigue syndrome, fibromyalgia, and multiple chemical sensitivities in a communitybased sample of persons with chronic fatigue syndrome-like symptoms. Psychosom Med. 2000;62:655-663.

4. Goldenberg DL, Simms RW, Geiger A, Komaroff AL. High frequency of fibromyalgia in patients with chronic fatigue seen in a primary care practice. Arthritis Rheum 1990;33:381-387.

5. Westert GP, Satariano WA, Schellevis FG, van der Bos GA. Patterns of comorbidity and the use of health services in the Dutch population. Eur J Public Health 2001;11:365372.

6. Struijs JN, Baan CA, Schellevis FG, Westert GP, van der Bos GA. Comorbidity in patients with diabetes mellitus: impact on medical health care utilization. BMC Health Serv Res. 2006;6:84.

7. Picavet HS, Hoeymans N. Health related quality of life in multiple musculoskeletal diseases: SF-36 and EQ-5D in the DMC3 study. Ann Rheum Dis. 2004;63:723-729.

8. Bollegala D, Perruccio AV, Badley EM. Combined impact of concomitant arthritis and back problems on health status: results from a nationally representative health survey. Arthritis Care Res (Hoboken). 2011;63:1584-1591.

9. El-Gabalawy R, Mackenzie CS, Shooshtari S et collab. Comorbid physical health conditions and anxiety disorders: a populationbased exploration of prevalence and health outcomes among older adults. Gen Hosp Psychiatry. 2011;33:556-564.

10. Moussavi S, Chatterji S, Verdes E, Tandon A, Patel V, Ustun B. Depression, chronic diseases, and decrements in health: results from the World Health Surveys. Lancet. 2007;370:851-858.

11. Creed FH, Tomenson B, Chew-Graham C et collab. Multiple somatic symptoms predict impaired health status in functional somatic syndromes. Int J Behav Med. 2013;20:194205.
12. Bombardier $\mathrm{CH}$, Buchwald D. Chronic fatigue, chronic fatigue syndrome, and fibromyalgia. Disability and health-care use. Med Care. 1996;34:924-930.

13. Scheeres K, Wensing M, Severens H, Adang E, Bleijenberg G. Determinants of health care use in chronic fatigue syndrome patients: a cross-sectional study. J Psychosom Res. 2008;65:39-46.

14. McNally JD, Matheson DA, Bakowsky VS. L'épidémiologie de la fibromyalgie autodéclarée au Canada. Maladies chroniques au Canada. 2006; 27:10-17.

15. Statistique Canada. Enquête sur la santé dans les collectivités canadiennes (ESCC) Composante annuelle. Guide de l'utilisateur: fichiers de microdonnées 2010 et 2009-2010 [en ligne]. Ottawa : Statistique Canada; 2011. Consultable en ligne à la page : http://www23.statcan.gc.ca/imdb-bmdi/pub /document/3226_D7_T9_V8-fra.htm

16. Deddens JA, Petersen MR, Lei X. Estimation of prevalence ratios when PROC GENMOD does not converge. SAS User Group International (SUGI) Proceedings, Seattle, Washington, March 30-April 2, 2003. Paper \#270-28.

17. Queiroz LP. Worldwide epidemiology of fibromyalgia. Curr Pain Headache Rep. 2013;17:356.

18. Collin SM, Crawley E, May MT, et collab. The impact of CFS/ME on employment and productivity in the UK: a cross-sectional study based on the CFS/ME national outcomes database. BMC Health Serv Res. 2011;11:217.

19. Reynolds KJ, Vernon SD, Bouchery E, Reeves WC. The economic impact of chronic fatigue syndrome. Cost Eff Resour Alloc. 2004;21:4.

20. Knight T, Schaefer C, Chandran A, Zlateva G, Winkelmann A, Perrot S. Healthresource use and costs associated with fibromyalgia in France, Germany, and the United States. Clinicoecon Outcomes Res. 2013;5:171-180.

21. Ursini F, Naty S, Grembiale RD. Fibromyalgia and obesity: the hidden link. Rheumatol Int. 2011;31:1403-1408. 
22. Goodwin L, White PD, Hotopf M, Standsfield CA, Clark C. Psychopathology and physical activity as predictors of chronic fatigue syndrome in the 1958 British birth cohort: a replication study of the 1946 and 1970 birth cohorts. Ann Epidemiol. 2011;21: 343-350.

23. Harvey SB, Wadsworth M, Wessely S, Hotopf M. Etiology of chronic fatigue syndrome: testing popular hypotheses using a national birth cohort study. Psychosom Med. 2008;70:488-495.

24. Dobkin PL, De Civita M, Bernatsky S, Kang H, Baron M. Does psychological vulnerability determine health-care utilization in fibromyalgia? Rheumatology (Oxford). 2003;42: 1324-1331.

25. Kriegsman DM, Penninx BW, van Eijk JT, Boeke AJ, Deeg DJ. Self-reports and general practitioner information on the presence of chronic diseases in community dwelling elderly. A study on the accuracy of patients' self-reports and on determinants of inaccuracy. J Clin Epidemiol. 1996;49:1407-1417. 